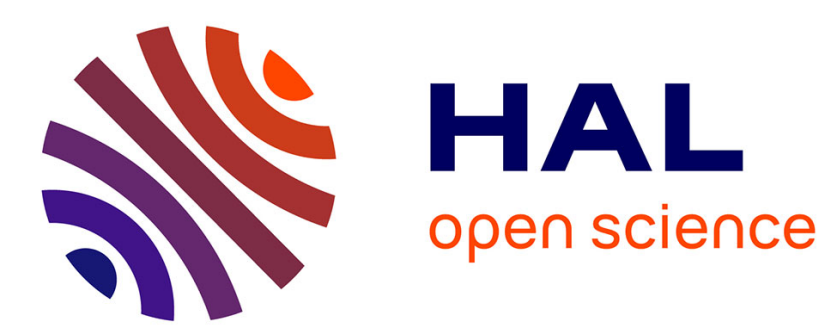

\title{
Éros politique. Idéologies du corps à la fin de l'Ancien Régime \\ Stéphanie Genand
}

\section{To cite this version:}

Stéphanie Genand. Éros politique. Idéologies du corps à la fin de l'Ancien Régime. Dix-Huitième Siècle, 2005, 37, pp.577 - 597. 10.3406/dhs.2005.2695 . hal-03405282

\section{HAL Id: hal-03405282 \\ https://u-bourgogne.hal.science/hal-03405282}

Submitted on 27 Oct 2021

HAL is a multi-disciplinary open access archive for the deposit and dissemination of scientific research documents, whether they are published or not. The documents may come from teaching and research institutions in France or abroad, or from public or private research centers.
L'archive ouverte pluridisciplinaire HAL, est destinée au dépôt et à la diffusion de documents scientifiques de niveau recherche, publiés ou non, émanant des établissements d'enseignement et de recherche français ou étrangers, des laboratoires publics ou privés. 


\section{Éros politique. Idéologies du corps à la fin de l'Ancien Régime} Stéphanie Genand

\section{Citer ce document / Cite this document :}

Genand Stéphanie. Éros politique. Idéologies du corps à la fin de l'Ancien Régime. In: Dix-huitième Siècle, $\mathrm{n}^{\circ} 37,2005$. Politiques et cultures des Lumières. pp. 577-597;

doi : https://doi.org/10.3406/dhs.2005.2695

https://www.persee.fr/doc/dhs_0070-6760_2005_num_37_1_2695

Fichier pdf généré le 23/09/2021 


\section{ÉROS POLITIQUE IDÉOLOGIES DU CORPS À LA FIN DE L'ANCIEN RÉGIME}

Lorsqu'il entreprend de définir le mot « corps » dans l'Encyclopédie, le chevalier de Jaucourt souligne d'emblée l'insaisissable polysémie du terme : «C'est une substance étendue et impénétrable, qui est purement passive d'elle-même, et indifférente au mouvement et au repos, mais capable de toute sorte de mouvement, de figure et de forme »'. Il désigne à la fois l'organisme et ses mouvements, les objets susceptibles d'entrer en contact avec nos sensations, et le rassemblement d'individus ou d'activités autorisés par le «Prince». Cet effort de taxinomie utilise en outre plusieurs comparaisons et métaphores, qui toutes essaient de cerner un domaine d'application de plus en plus vaste: «L'anatomie étant une espèce de géographie dans laquelle la précision est nécessaire, on a divisé le corps, comme la terre, en plusieurs régions ${ }^{2}$. Objet paradoxal, le corps finit même par se dérober au sens, comme le confessent les dernières lignes de l'article, renvoyé à son impuissance : «Quoique nous ayons rapporté un grand nombre d'acceptions différentes du mot corps, nous ne nous flattons pas de n'en avoir omis aucune ; mais celles qui précèdent suffisent pour donner une idée de l'étendue dans la langue, de ce mot qui désigne une chose qui en a tant dans la nature $\gg$.

Le $18^{\mathrm{e}}$ siècle trouve donc dans le corps un domaine tout à la fois de savoir et d'ignorance, de lumière et d'obscurité. Cette ambiguïté s'explique par un double mouvement contradictoire : les progrès scientifiques permettent un afflux de connaissances nouvelles, en matière de médecine et d'anatomic notamment. Mais ils n'empêchent pas l'extension métaphorique de plus en

1. Encyclopédie ou Dictionnaire raisonné des sciences, des arts et des métiers, nouvelle impression en fac-similé de la première édition de 1751-1780, StuttgartBad Cannstat, Frommann Verlag, 1988, vol. 4, p. 261.

2. Ibid., p. 264.

3. Ibid., p. 269. 
plus importante qui s'empare du mot: le corps désigne aussi bien la physiologie humaine que l'organisation sociale et politique, selon le principe déjà très ancien de l'homologie entre microcosme et macrocosme.

Ce réseau d'images, s'il ne date pas du $18^{\mathrm{c}}$ siècle, connaît cependant une extension nouvelle à la veille de la Révolution. Les travaux d'Antoine de Baecque ${ }^{4}$ ont permis de mettre en lumière la récurrence des images corporelles dans la vie politique au tournant des Lumières. La montée des valeurs bourgeoises, à partir de 1780, attise en effet la violence contre les élites dirigeantes, coupables aussi bien dans leurs privilèges que dans leurs mœurs. Cette pression idéologique, accrue dans les dernières années de l'Ancien Régime, accélère le transfert métaphorique entre deux corps : celui du libertin, confiné dans les plaisirs de boudoirs, et celui de l'aristocratie, hostile à toute refonte du système. Cette lecture apparaît bien avant la Révolution, au sein de textes «mondains » encore largement inspirés du modèle de Crébillon. Plusieurs récits de séduction, qui reprennent la trame et les personnages du libertinage des années 1730, rompent effectivement avec la tradition « autarcique » d'une écriture coupée du monde extérieur. L'argent, les conditions matérielles des personnages, les événements politiques et les aléas du pouvoir, forcent les portes du boudoir: le libertinage, à la fin du siècle, s'ouvre au temps qui passe et au mouvement plus général de l'histoire. Dans cette optique, la représentation du corps se charge elle aussi de nouvelles valeurs. L'heure n'est plus aux conquêtes exclusivement cérébrales. Il faut dévoiler la séduction sous son jour le plus cru, moins pour exciter le lecteur que pour dénoncer, à travers les jeux physiques, la crise d'un système tout entier. La représentation du corps, dans un tel contexte, devient un enjeu inédit. Elle transmet en filigrane, derrière le plaisir érotique, un message de plus en plus marqué : il faut, à tous les niveaux, renouveler des corps aussi indécents qu'obsolètes.

Avant d'être explicitement politique, c'est-à-dire engagé dans les mécanismes du gouvernement, le corps représente, au tournant des Lumières, un signe idéologique. L'excitation du lecteur importe moins désormais que sa mise en garde: le libertinage est une menace pour la société française. Cette condamnation morale, récurrente à partir de 1780 , s'appuie sur la représentation

4. Voir notamment Le Corps de l'histoire, Métaphores et politiques, 17701800 (Paris, Calmann-Lévy, 1993). 
du corps : rien ne vaut, pour convaincre le public, une mise en scène de la débauche. Le transfert métaphorique fonctionne alors pleinement : le libertin paie dans sa chair le prix de ses frasques d'aristocrate. À corps social coupable, biologie corrompue. Ainsi Joseph Maimieux, lorsqu'il publie en 1788 Le Comte de Saint Méran ou les nouveaux égarements du cour et de l'esprit, orientet-il sa suite du roman de Crébillon dans un sens beaucoup plus sombre. Si l'initiation de Meilcour restait inachevée, et limitée aux dévoilements érotiques du novice, celle de son neveu Germeuil va plus loin. Le personnage ne découvre plus seulement les boudoirs, mais leurs coulisses sordides et effrayantes :

Les lambris étaient chargés de petits sujets en camaïeu, et les encoignures de groupes de biscuits sous glace, qui réunissaient tout ce que peut se figurer l'imagination la plus lascive ; et le goût exquis et la rareté des mets ajoutaient encore leur amorce à celle des autres voluptés. Mais l'appétit manquait à presque tous les convives, et ces amateurs excédés avaient presque tous la vue basse ou très affaiblie. Des impatiences de nerfs, des fibres relâchées ou desséchées, des esprits appauvris, des maux palliés, des papilles brûlées, des tympans racornis, des sens blasés par un abus continuel, émoussaient la pointe trop déliée pour eux de ces délices outrés [...]. Bientôt l'obscène équivoque, le calembour, la calomnie, des assaisonnements stimulants, des liqueurs spiritueuses et incendiaires donnent une élasticité artificielle à des facultés en stupeur et les agaceries de la lubricité portent jusqu'au délire l'exaltation de la débauche. ${ }^{5}$

Le texte utilise tous les ressorts du libertinage mondain : le type de personnages, le schéma de l'initiation sensuelle, le milieu de la noblesse française. Pourtant, l'éducation de Germeuil, si elle commence comme celle de Meilcour, place vite la représentation de la séduction sous le signe de la surenchère. À l'image de cette scène, les soirées galantes souffrent de leurs excès : que reste-t-il, dans ce tableau, des égarements du cœur et de l'esprit ? Le décor est saturé d'objets du plus mauvais goût, les plats tentent, à force d'épices, de réveiller les sens blasés des convives, et les corps semblent des mécaniques enrayées : les organes ont perdu leur acuité, les humeurs ont séché, et l'esprit lui-même, jadis si prompt à saisir l'occasion d'un bon mot, s'est empêtré. La représentation se transforme en réquisitoire, et l'érotisme cède sous la pression des valeurs de plus en plus négatives qui pèsent sur le corps. De séduisant, il devient, à la fin du siècle, repoussant.

5. Joseph Maimieux, Le Comte de Saint-Méran ou les nouveaux égarements du cour et de l'esprit (Londres, Paris, Leroy, 1788), t. 3, p. 142. 
Ce qui se dessine même, à la veille de la Révolution, c'est une pathologie du corps libertin. Malade, usé, déréglé, il traduit en signes cliniques la menace qui pèse sur la séduction. Lorsqu'il publie en 1789 Le Danger des circonstances, Nougaret s'inspire de la trame des Liaisons dangereuses : comme Laclos, il retrace l'histoire d'un complot, organisé non contre une femme mais contre deux frères par un libertin du nom de Fauxfilter. Les chevaliers de Joinville suivent à Paris leur éducation mondaine et militaire, mais leur mère redoute pour eux le danger des mauvaises fréquentations. Le titre de Laclos informe donc la structure de ce texte, qui donne cependant un visage concret aux « liaisons dangereuses ». La métaphore, suggestive en 1782, s'incarne chez Nougaret. La « liaison dangereuse »se repère aux stigmates qui la marquent :

Les jeunes gens s'accoutument facilement au désordre; les plaisirs criminels qu'ils peuvent se procurer dès qu'ils les désirent, leur paraissent préférables à ceux que la raison approuve, et qu'il faut acheter par une longue attente. Il en résulte qu'habitués à ne vivre qu'avec des personnes vicieuses, ils sont très déplacés dans une société choisie, et contractent une timidité, des manières et un langage qui ne font l'éloge ni de leur esprit ni de leur éducation. J'en ai vu quelques-uns de ces malheureux libertins dans le monde; il est aisé de les connaître au peu de fraîcheur de leur teint, au délabrement de leur santé. On marie ces êtres déjà presque nuls pour le bien général, auquel chacun doit contribuer par ses talents et son industrie ; ils corrompent souvent la santé de leur infortunée compagne, et donnent le jour à des enfants infirmes, qui traînent dans les douleurs une longue et pénible existence. ${ }^{6}$

Le corps véhicule ici une véritable pathologie: individuelle, puisque le séducteur n'offre plus qu'un visage rongé par la maladie, et collective puisqu'il perd sa fertilité. Usé, énervé au sens étymologique, le libertin représente ainsi un double fléau pour la collectivité. Il en dévore les richesses en parasite social, et compromet son avenir en menaçant le renouvellement de la nation. Avant même la Révolution qui s'empare avec force de cette idée, les récits de séduction entretiennent donc le mythe d'une noblesse épuisée à force de débauches. Les mémoires de courtisanes, qui fleurissent à la fin du siècle, s'étaient déjà fait le relais de cette image : les prostituées, spectatrices privilégiées de l'évolution des pratiques, témoignent de la corruption montante

6. Nougaret, Le Danger des circonstances, ou lettres du chevalier de Joinville et de mademoiselle d'Arans (Bruxelles, veuve Dujardin; Paris, Defer Demaisonneuve, 1789), t. 2, p. 223. 
et de la généralisation des goûts «baroques ». En 1784, La Correspondance d'Eulalie mentionne dans plusieurs de ses lettres les exigences de plus en plus perverses des clients :

Que les hommes, ma chère amie, ont des goûts bizarres! Hier, chez la Présidente, il m'a fallu fouetter pendant plus de deux heures un vieux président, tandis qu'à genoux devant moi il me gamahuchait.

On a bien raison de dire, Minette, que les goûts des hommes dans leurs jouissances sont encore plus fantasques que les caprices de leurs caractères. L'amour en gémit, mais il excuse tout. Une fois adonné au culte du libertinage, il faut savoir s'y prêter. Je me vois journellement obligée d'apprendre de nouvelles fantaisies.

Avoue, mon cœur, qu'il y a des hommes qui ont des goûts bien baroques, et auxquels il est impossible de rien concevoir. Il serait à souhaiter que $M$. le comte de Buffon, si célèbre naturaliste, voulût nous en donner l'explication. ${ }^{7}$

Ces jeux de l'amour, derrière l'anecdote piquante, fonctionnent surtout comme des signes. Signes d'une virilité en crise, et d'une déperdition des énergies. Un autre « témoignage » publié la même année, Les Confessions d'une courtisane devenue philosophe, renforce cette idée de contamination entre le domaine public et la sphère privée. La narratrice retrace l'histoire de sa déchéance, tout en liant cette chute au parcours du pays dans son ensemble :

Chaque désordre particulier forme nécessairement la masse du désordre général, et la masse du désordre général contribue ensuite au désordre particulier; de sorte qu'ils agissent l'un par l'autre, et se propagent à l'infini. Les caractères s'énervent, les constitutions s'affaiblissent, les empires deviennent chancelants; et lorsque la corruption est parvenue au dernier période, que l'édifice moral est, pour ainsi dire, renversé, il survient une crise, une révolution qui détruit ou rétablit entièrement le corps politique. ${ }^{8}$

La métaphore centrale, qui permet la contagion ou, pour reprendre le terme du texte, la fermentation, reste donc celle du corps : corps politique, conformément à l'étymologie complexe du terme, et corps-organisme, menacé de corruption, de maladie ou de dégénérescence. Dans cette optique clairement idéologique, la

7. Correspondance d'Eulalie, ou tableau du libertinage de Paris, avec la vie de plusieurs filles célèbres de ce siècle, 1784, réed. in CEuvres anonymes du XVIII siècle, «L'Enfer de la B.N. » (Paris, Fayard, 1986), t. 4, p. 70, 249 et 106.

8. Les Confessions d'une courtisane devenue philosophe (Londres, Paris, Couturier, 1784), Avant-propos, p. I. 
vie des courtisanes se coupe du plaisir et de toute forme de gaieté :

Elles ne connaissent ni l'amour, ni l'amitié : leurs organes sont usés, et l'expression même du plaisir est presque toujours grimacée : elles paraissent liées entre elles, et se détestent : jalouses de celles qui ont de plus beaux ajustements, qui paraissent avec plus d'éclat, elles se liguent pour les déchirer, et nuire à leur bien-être : elles n'ont pas de plus grande satisfaction que de les voir retomber dans l'humiliation et dans la poussière, dont elles étaient sorties. C'est pourtant à de pareils objets qu'une partie des hommes sacrifie la santé, la fortune et la réputation. C'est dans ces lieux de débauche, où résident les vices les plus infâmes, qu'ils vont passer leur vie, renonçant aux sociétés honnêtes, où ils trouveraient également à satisfaire le cœur et l'esprit, et abandonnant quelquefois des épouses et des enfants auxquels ils refusent des choses indispensables, tandis que, d'un autre côté, ils donnent avec profusion des choses superflucs. ${ }^{9}$

La décadence du corps s'observe donc à tous les stades de l'existence, du plus intime - la manifestation du plaisir sexuel au plus affiché - les institutions politiques qui régissent la cité.

La Révolution marque une étape décisive dans le durcissement de cette métaphore : à partir de 1789 , le corps devient une arme politique au service du régime. Tout était prêt, dans les textes, pour l'émergence d'une telle propagande : le sentiment d'une crise imminente, d'un essoufflement, et la désignation d'une liste de suspects dénoncés par la mise en scène de leur corps. La radicalisation révolutionnaire, accompagnée de la liberté d'expression proclamée depuis peu, s'empare de cette structure avec force : l'aristocrate devient l'ennemi officiel du régime, et le libertinage la marque d'une appartenance coupable à l'Ancien Régime. Seules la virulence des attaques et la puissance unilatérale du message changent après 1789. Le mythe qui s'impose alors, comme l'ont montré les analyses d'Antoine de Baecque, est celui de la "régénération »: renouveau des organismes mais aussi des structures politiques, elle désigne d'un seul mouvement la refonte de tous les corps dont rêvent les révolutionnaires ${ }^{10}$.

9. Ibid., p. 62 .

10. "La langue de la régénération pose ainsi la métaphore corporelle. le "corpsrécit", au centre des croyances et des écrits politiques : la France révolutionnée ne serait-elle pas l'image d'un corps prêt à retrouver sa vitalité originelle, prêt à se relever de cette maladie de langueur qu'ont décrite les écrivains politiques de la fin de l'Ancien Régime? Cette politisation du concept de régénération. évidente dans les mots de 1789, est cependant récente. En effet, avant 1730, la régénération appartient encore aux seuls vocabulaires religieux et médical " ( $L e$ Corps de l'histoire, op. cit., p. 165). 
La récupération politique de ce concept, jusqu'ici réservé aux domaines de la religion et de la médecine, est indissociable des événements. C'est la convocation des États généraux qui semble accélérer la vision du pays comme un organisme malade qu'il faut soigner au plus vite ${ }^{11}$. De quoi souffre alors la France ? De la «maladie d'Ancien Régime » : les élites qui accaparaient les forces de la nation sont corrompues, usées, malades. La noblesse est accusée de maintenir l'illusion passéiste du libertinage, et de gaspiller son énergie dans les seuls combats des sofas. À l'heure des affrontements politiques décisifs, le plaisir, aussi gratuit qu'égoïste, est un crime de lèse-révolution. Parmi le nombre impressionnant de pamphlets qui fleurissent alors sur le sujet, Les Délices de Coblentz offre un exemple significatif. I es émigrés réfugiés en Allemagne autour du prince de Condé s'illustrent d'abord par une faute collective, une faute de corps social: nier le passage du temps et l'abolition de l'Ancien Régime :

On ne voit que des fêtes, des orgies et des rendez-vous de galanterie. Il semble que les Français soient le peuple de la terre le plus enclin aux libres jouissances, et les Bourbons, qui, maintenant, enrichissent cette contrée de l'Allemagne, peuvent dire, après avoir quitté les délices de Paris, comme dit Sertorius, dans une des plus belles tragédies du grand Corneille : Rome n'est plus dans Rome, elle est toute où je suis. ${ }^{12}$

Cette reconstitution factice, facilitée par les fortunes emportées outre-Rhin, est une première amnésie du fait révolutionnaire : le passé n'est pas mort pour qui se donne les moyens de sa résurrection. Elle se prolonge dans le culte de la jouissance célébrée tous les jours à Coblentz. Comme le titre l'indique, tout n'y est que délices, et le plaisir finit par détrôner le projet de la contrerévolution. L'émigration s'accompagne donc d'une disparition de la réalité politique : la Révolution n'existe plus, ni avec elle la mise hors-la-loi du corps aristocratique. Le groupe peut alors s'abandonner au luxe des conquêtes sexuelles, selon le principe de la contagion du public et du privé :

Vous observez sans doute, que nous autres femmes, nous ne nous mêlons point de projets politiques. Si nous faisons la guerre, ce n'est que sur des canapés et la verdure. C'est à coups de cul que nous militons et je puis vous assurer que nous avons souvent l'avantage sur nos

11. Ibid., p. 169.

12. Les Délices de Coblentz ou Anecdotes libertines des émigrés français (Coblentz, 1792), p. 8. 
agresseurs qui nous demandent quartier. Nous sommes bien obligées de le leur accorder, mais nous avons soin de stipuler avec eux, que nous ne consentons qu'à une trêve de peu de durée ; qui suffit néanmoins pour leur laisser la faculté de reprendre leurs forces et de revenir à l'assaut. [p. 71]

Or, c'est précisément dans cette mise en avant du corps, social et érotique, que réside la faute de l'aristocratie. Le message politique impose alors au fil des pages un durcissement du ton, et un portrait du noble en organisme dégénéré :

Je n'avais point encore l'idée de la magnificence de ces Sardanapales français, des Satrapes royaux, qui, émoussés, usés, énervés jusqu'à l'impuissance, n'ont conservé que le goût du plaisir et de violents désirs. Tout épuisés qu'ils sont, et incapables de jouir naturellement, l'habitude ancienne et perpétuellement renouvelée de se vautrer dans les sentines de l'impureté, leur fait un besoin de s'y replonger sans cesse. C'est alors que, pour ressentir de nouveaux attraits dans les excès vénériens, il est indispensable d'avoir recours à des artifices, à des enchantements surnaturels, qui achèvent de brûler, de consumer le corps humain, d'en dessécher les muscles, d'en appauvrir le sang, d'en affaiblir les ressorts, au point que la machine, une fois démontée, calcinée et détruite, n'offre plus, sur sa figure, que le triste spectacle d'un squelette hideux. [ibid.]

On retrouve dans ce tableau les tares mentionnées dans les romans de la fin du siècle: perte des énergies, dessèchement des organes, disparition de toute forme de muscle. Le corps aristocratique est une biologie rouillée au service d'une classe qui se délite :

Notre comité féminin était composé de femmes de tout étage. Les madames avaient trop oublié leur dignité pour en conserver, en ce moment, le plus léger souvenir, et leurs propos les plus doux imitaient ceux des harengères bricolées dans un taudis par des portefaix ou des Auvergnats. Leurs compagnons, flattés d'être préférés à leurs maîtres, quoiqu'un tel avantage arrive souvent à ceux de leur espèce, travaillaient chaudement, pour ne pas se rendre indignes des faveurs de leurs amoureuses maîtresses. ${ }^{13}$

Cette dénonciation du corps noble s'inscrit dans un vaste mouvement de prophylaxie qui se renforce au moment de la Révolution. Les réflexions hygiénistes s'étaient multipliées à partir des années 1760-1770: dans la lignée des recherches médicales de Tissot ${ }^{14}$, Rétif propose de légiférer sur le système de la prostitu-

13. Suite des Délices de Coblent:, op. cit., p. 49.

14. Tissot, L'Onanisme, dissertation sur les maladies produites par la masturbation (Lausanne, 1760). 
tion dans son Pornographe ${ }^{15}$ en 1769 : la fréquentation des courtisanes, comme la pratique de l'onanisme, dilapide la semence et ralentit l'essor démographique. Cette angoisse, récurrente dans la seconde moitié du siècle, s'accélère avec la Révolution : le changement de régime a besoin de s'appuyer sur des bataillons toujours plus nombreux de jeunes patriotes. Il faut à la France régénérée une population dynamique, à la hauteur du bouleversement accompli. Un glissement s'opère alors entre ce qui relevait du strict domaine de la morale et ce qui bascule, après 1789 , dans la sphère politique. Là où Diderot, dans les Salons de 1767 , reprochait à Baudouin son incitation à la débauche ${ }^{16}$, les pamphlétaires accusent l'aristocrate de compromettre la refonte du système. La faute n'est plus seulement éthique, elle devient politique. Plusieurs textes s'emparent alors de ce support hygiéniste pour dénoncer ce qui apparaît comme une trahison de la noblesse. Dom Bougre aux États-Généraux reprend en 1789 les propositions avancées par le même Rétif vingt ans plus tôt :

Le remède à tous ces abus serait, je crois, d'adopter le système de feu sieur de la Bretonne, grand écrivain moraliste. Il a proposé, dans son ouvrage intitulé Le Pornographe, de chasser toutes les filles de joie, de les diviser en différentes maisons. Les prix différents seraient gravés sur la porte d'entrée... Il est certain que ce plan, outre qu'il éviterait tous les inconvénients dont nous avons fait mention, faciliterait la visite et la guérison des corps malades, qui seraient mis dans la chambre des branleuses. ${ }^{17}$

Il faut, au plus vite, réduire le gaspillage de la fertilité, ce qui passe par une rationalisation de la prostitution et par un classement des pratiques sexuelles. Ne devront avoir cours que celles qui permettent la procréation, au détriment de la seule recherche du plaisir :

15. Rétif de la Bretonne, Le Pornographe, ou Idées d'un honnête homme sur un projet de règlement pour les prostituées, propre à prévenir les malheurs qu'occasionne le publicisme des femmes (Londres, 1769).

16. « Artistes, si vous êtes jaloux de la durée de vos ouvrages, je vous conseille de vous en tenir aux sujets honnêtes. Tout ce qui prêche aux hommes la dépravation est fait pour être détruit. [...] Je ne puis me dissimuler qu'un mauvais livre, une estampe malhonnête que le hasard offrirait à ma fille, suffirait pour la faire rêver et la perdre ", Diderot, Euvres esthétiques (Paris, Garnier, 1976), p. 472473.

17. Rétif de la Bretonne, Dom B... aux États-Généraux, ou Doléances du portier des Chartreux, par l'auteur de la Foutromanie, à Foutropolis, chez Braquemart, libraire, rue du Tire-vit à la couille d'or, avec permission des supérieurs, in CEuvres érotiques de Rétif de la Bretonne, "L'Enfer de la B. N. » (Paris, Fayard, 1985), t. 2, p. 551. 
Je viens ensuite, Messieurs, vous apprendre les moyens d'obvier à ces profanations, d'épurer les mœurs, de prévenir l'abâtardissement de la race humaine, de détruire l'adultère, la sodomie, la bestialité et autres vices qui dégradent les Français depuis cinq à six générations. [p. 548]

De ce catalogue des postures « utiles » à la condamnation de leurs adeptes, il n'y a qu'un pas, que les pamphlétaires franchissent assez vite. L'aristocrate n'est plus seulement dénoncé comme corps dégénéré, comme classe coupable, il l'est aussi comme partisan d'une érotique à la fois décadente et narcissique :

Vous verrez, Messieurs, qu'il ne faudra pas plus de vingt séances pour faire une loi qui ramène les hommes et les femmes à foutre tout bonnement, pour faire des enfants et se dégorger les reins. Ô garces [...] qui m'avez gâté le tempérament; garces à sentiment, qui m'avez corrompu le cœur ; garces à argent, qui avez ruiné ma bourse ; garces de toute espèce, tant mâles que femelles, votre règne va finir : ce n'est pas du foutre, c'est du sang que la nation verse dans cette grande époque. [ibid.]

Parmi les pratiques sexuelles dénoncées comme « improductives », la sodomie occupe une place privilégiée. Apanage des bougres et des moines pendant tout le dix-huitième siècle, elle devient une cible politique après 1789 : elle ne sert pas le renouveau démographique, et entretient la spécificité de ce clergé dont les révolutionnaires ont fait leur ennemi après la déchristianisation du pays. Le topos du prêtre sodomite, fer de lance de la littérature «philosophique », passe ainsi du registre érotique à celui de la propagande politique : la matière n'est pas nouvelle, qui recycle les thèmes usés de Dom Bougre portier des Chartreux et de Thérèse philosophe, pour ne citer que les plus connus des chefsd'œuvre du genre. Ce qui change en revanche, c'est la posture énonciative. L'heure n'est plus à la connivence érotique avec le lecteur, mais à la délation sous les feux de la tribune. Suivant en cela le mouvement de la Révolution, qui produit à satiété des décrets et des textes de loi, les pamphlets confondent érotique et politique : plus que jamais, le corps est au croisement des métaphores. Les prêtres sont ainsi présentés, eux aussi, comme des organismes épuisés :

Vous les voyez paraître, le front ceint d'une vieille peau de zibeline, de chat ou de mouton; les uns avec des visages rubiconds et enluminés d'un rouge incarnat, surchargés de dartres, de boutons et d'échauffures ; les autres, sous les traits livides d'une indolente momie, les yeux mornes et caverneux, les paupières allongées, les joues creuses, la langue surchargée de vapeurs méphitiques, les dents décharnées, le virus prêt à expirer sur les lèvres, la démarche incertaine et chancelante, le buste courbé, 
les épaules hautes et la tête baissée ; enfin, avec tous les symptômes de la débauche et de l'incontinence, de la lubricité et des maladies qui en résultent. C'est sous ses masques hideux que, chaque jour, appelés par leur état, ils vont dans le sanctuaire de la divinité porter indolemment les restes dégoûtants d'un corps cacochyme, et plein de défectuosités. ${ }^{18}$

Un changement de ton spectaculaire s'est opéré en 1790. Si le bougre attirait auparavant la sympathie inspirée par les figures d'un patrimoine commun, il devient sous la Révolution un ennemi du régime. Faut-il lire ces réquisitoires comme des dénonciations sincères, ou des parodies des décrets qui s'emparent de tous les domaines après 1789 ? Les deux dimensions se recoupent probablement : la Révolution nourrit les angoisses démographiques, légifère pour cela sur les pratiques sexuelles, mais les pamphlétaires relaient autant qu'ils en jouent sa soif de contrôle. La Requête et décret en faveur des putains, des fouteuses, des maquerelles et des branleuses contre les bougres, les bardaches et les brûleurs de paillasse, offre un exemple assez troublant de cet entrelacs d'intentions. Ce texte illustre au premier regard, comme l'indique son titre, une politisation de la sexualité : une pratique érotique, la sodomie, fait l'objet d'un décret qui ordonne de lui préférer la fréquentation des courtisanes. Le destinataire, le «bougre », est désormais un mauvais patriote : il dilapide sa semence et ralentit la régénération nationale. Il fait donc l'objet d'une remontrance officielle, qui se moule dans la rhétorique du texte de loi :

Sur cette requête, dont le Comité de Fouterie a reconnu la justice, les fouteurs nationaux ont rendu un arrêt qui fait droit aux fins requises, revêtu de toutes les formalités nécessaires et scellé, pour la plus grande authenticité, du plus grand Sceau de la Nation portant l'empreinte d'un vit majestueux barbouillant de foutre les lèvres vermeilles d'un con couronné d'une guirlande de couilles.

Décret : Nous, restaurateurs de la France, / Au Manège tenant séance, / Faisant droit aux Conclusions, / Comme il est requis, ordonnons / Que tout marchand de cristalline, / Ayant pour plumet une pine, / Porte écrit au milieu du front, / En caractères gros-canon / Pour que de loin on le devine, / JE SUIS RAMASSEUR DE MARRONS. ${ }^{19}$

18. Les Travaux d'Hercule, ou La Rocambole de la fouterie, par un émule de Piron, Grécourt et Gervais (Paris, l'an deuxième de la liberté), in Patrick Wald Lasowski, La Science pratique de l'amour, Manuels révolutionnaires érotiques (Paris, Philippe Picquier, 1998), p. 212.

19. Requête et décret en faveur des putains, des fouteuses, des maquerelles et des branleuses contre les bougres, les bardaches et les brûleurs de paillasse, à Gamahuchon, et se trouve chez toutes les fouteuses nationales, l'an second de la régénération foutative, in CEuvres anonymes du XVIII' siècle, «L'Enfer de la B.N. " (Paris, Fayard, 1987), t. 6, p. 387. 
Mais le mélange des registres, de l'officiel grandiloquent à l'ordurier, laisse évidemment à penser que cette Requête relève autant de la parodie que du réquisitoire. La parole de l'Assemblée, écrite en langage érotique, comme le contraste entre la crudité du vocabulaire et la sécheresse de l'injonction, vont dans le même sens : la confusion des genres rend le texte insaisissable, et invalide en partie la portée de son message.

Cette politisation de la sexualité se retrouve dans la plupart des pamphlets, et dépasse la figure du «bougre ». La Révolution condamne plus généralement tous les signes de raffinement, de lenteur et d'érotisme. Les révolutionnaires ne valorisent pas seulement la "régénération », ils soulignent aussi les vertus de la rapidité, de la force et de la virilité. La nation en marche a besoin de patriotes nombreux et solides. Dans un tel contexte, les " gradations » sont forcément contre-révolutionnaires. L'arsenal des textes libertins, leurs personnages, leurs femmes à l'ottomane, leurs courtisanes, leurs postures faussement pudiques, se voit donc chargé d'une mission nouvelle : dénoncer cet art de vivre comme une éthique décadente. Ce qui change, à la lecture de ce déjà-vu, c'est la nature du regard : le réquisitoire a remplacé la connivence. L'Écho Foutromane, publié en 1792, offre un exemple frappant de cette nouvelle optique. L'auteur ressuscite la plupart des topoï libertins : un jeune homme est initié aux plaisirs de l'amour par plusieurs courtisanes, et passe en revue, au fil des pages, les différents types de séductrices capables de former un novice. La première étape de son parcours l'amène donc chez une femme qui dissimule son «appétit » sous un jeu de postures :

Elle est sensible par tempérament et même par sentiment ; un sourire, un coup d'œil, sont les mots du guet qui portent en substance que l'on peut aspirer à elle, pourvu qu'on observe de très grands ménagements ; et ces ménagements demandent que vous osiez, pour ainsi dire, à son insu. Il faut, à cet effet, que le hasard ou quelque prétexte adroitement amené, vous ait conduit chez elle; il faut, s'il se peut, que vous la trouviez mollement étendue sur un sofa, vêtue à la légère, la gorge recouverte d'une simple gaze ; il serait mieux que dans cet état ou à peu près, elle vous eût donné rendez-vous dans son boudoir, et qu'clle cût l'air de l'avoir oublié. Vous y pénétrerez en quelque sorte furtivement ; et pour sauver à sa pudeur la honte d'une défaite, elle sommeillera précisément à l'instant où vous arriverez. ${ }^{20}$

20. L'Écho foutromane, ou recueil de plusieurs scènes lubriques ou libertines, contenant les épreuves de l'abbé Dru, le secret de madame Conléché, l'entrevue de mademoiselle Pinelli avec Arlequin et Pierrot, la solitude de madame Convergeais, etc. Sur l'imprimé à Démocratis, aux dépens des fouteurs démagogues, 1792, p. 7. 
Cette description garde, en 1792, quelque chose d'artificiel : on se croirait, à la lire, dans un tableau de Boucher. Mais ce cliché a désormais un nouvel objectif : servir de faire-valoir à la femme patriote. L'Écho foutromane oppose en effet, quelques pages plus loin, cette coquette hypocrite à la jouisseuse moderne :

On fera paraître sur la scène, tantôt une ribaude qui s'énoncera et agira selon le caractère qu'on aura eu la précaution de lui dessiner, tantôt ce sera une ci-devant... qui fera sa petite bouche, et la prude dans sa manière de rendre sa pensée concupiscive, et n'en croquera pas moins une grosse andouille dans sa partie passablement ouverte. [p. 9]

La «ribaude », version patriote de la femme libertine, n'a pas peur ni honte de son désir : franc, rapide, vigoureux, il n'a plus besoin de se voiler sous une quelconque gaze. Ce texte confronte ainsi, derrière ces figures féminines, deux érotiques différentes : les gradations coupables et la fougue du désir. La célèbre « lettre à l'écritoire » des Liaisons dangereuses ${ }^{21}$ où Valmont utilise le corps d'Émilie pour écrire à Madame de Tourvel, fait l'objet du même travail d'explicitation :

Madame Connillac se trousse aussitôt et relève sa chemise jusqu'audessus des reins ; l'abbé Dru prend une feuille de papier, l'applique sur la fesse droite et y écrit son refus. - Tu me chatouilles, vilain abbé, s'écriait de temps en temps madame Connillac. Et il continue de se rendre réfractaire par le refus qu'il y' trace. Dès qu'il a fini, il baise l'un et l'autre côté de son adorable pupitre, et remet son bulletin à madame Connillac. [p. 28]

La situation d'énonciation, source de l'ambiguïté chez Laclos, se transforme ici en scène sexuelle: tout est fait pour que le corps des personnages, dont les patronymes soulignent le goût pour la jouissance directe, transparaisse sous la lettre. À sexualité libertine, littérature contre-révolutionnaire, pourrait-on dire pour résumer la propagande de l'époque. À l'inverse, une corrélation directe semble s'établir entre l'énergie du désir et la force politique : si la libertine trahit la nation par ses lenteurs, le révolutionnaire saura satisfaire d'un même élan et sa femme et le nouveau régime. L'Écho foutromane établit un parallèle explicite entre ces deux énergies ; un jeune soldat blessé lors de la prise de la Bastille peut, malgré sa mutilation, offrir la meilleure nuit de noces à l'épouse qui l'attend :

21. Laclos, Les Liaisons dangereuses, lettre XLVIII (Paris, Gallimard, 1979), p. 99-100. 
Cet époux jeune et ardent s'était transporté dans les huit tours de la Bastille, et content d'abattre le despotisme avant de combattre un pucelage, il voulait, en franc patriote, mêler quelques branches de laurier à la couronne de myrte que l'amour lui préparait dans les bras de sa femme ; son patriotisme ne fut pas récompensé : un plomb meurtrier, vomi par une bouche infernale, vint frapper ce malheureux, précisément au-dessous du bas-ventre ; et lui enlevant ses deux testicules, ne lui laisse que la moitié d'un vit qui, cette nuit même, devait tout seul opérer une révolution d'une autre espèce en changeant une fille en femme. Il ne respire un instant dans cet état déplorable que pour gémir sur son épouse et pour pester contre l'aristocratie qui, plutôt que de le dégrader de la sorte, n'avait pas préféré de lui percer le cœur. [p. 36]

Celui qui a vaincu le despotisme dispose d'une puissance telle qu'il saura se montrer, dans toutes les circonstances, un amant fougueux ; capable en tous cas de réussir, après la « Révolution » majuscule, la «révolution» intime de la défloration. Le texte suggère même un jeu de mots autour de la notion de "sansculottes $\gg$ : prise au sens propre, la métaphore désigne ici, en plus de l'appartenance populaire et de la simplicité vestimentaire, la force érotique. Claude Mazauric définit ce terme, dans le Dictionnaire historique de la Révolution française, comme le croisement d'un sens social et politique ${ }^{22}$. En 1792, en pleine période de revalorisation du mot, $L$ 'Écho foutromane ajoute apparemment un sens sexuel à l'image : frappé «au-dessous du basventre », le héros de la Bastille perd sa culotte mais pas pour autant sa virilité ni son énergie. La gloire politique du «sansculotte » transcende ainsi les limites de son physique et l'emmène dans un corps idéal, sublime : le corps du héros de la Révolution.

Ce dernier s'incarne même dans une figure phare à la fin du siècle: Hercule. Le régime révolutionnaire ne se contente pas

22. «Employé dans les premières années de la Révolution par les publicistes anti-révolutionnaires, le désignant "sans-culottes" se veut injurieux. Vers mars 1791, dans la presse royaliste à destination populaire comme Le Journal de la Cour et de la Ville, le syntagme "sans-culottes" sert à désigner la horde des dćgucnillés et des désargentés qui errent autour du Palais-Royal, et fréquentent les députés de gauche ; ces gens "montrent leur cul à travers leurs guenilles". "Sans-culottes" remplace "canaille" qui fait désuet. Par l'absence remarquée de la culotte, on veut à l'évidence distinguer en même temps que la pauvreté, la bestialité, l'inculture et la nudité grossière. Mais le vocabulaire de la lutte des classes, tendanciellement bipolaire, en opposant "sans-culottes" à "aristocrates" favorisera peu à peu son identification à "peuple". Dès lors, le retournement de l'injure en titre de gloire [...] s'effectuera de manière symétriquement inverse à la dévalorisation du mot "aristocrate" puis "modérés" ", dans Albert Soboul (dir.), Dictionnaire historique de la Révolution française (Paris, P.U.F., 1989), p. 957. 
de dénoncer la sexualité de l'Ancien Régime et ses protagonistes, il prétend former le citoyen et lui enseigner les meilleures manières d'utiliser son corps. Une pédagogie érotique se développe ainsi à partir de 1790 . Ce projet de refonte suppose une littérature spécifique, qui place l'éducation avant le plaisir de l'intrigue ou la construction des personnages. La période voit alors se multiplier les «bréviaires du parfait patriote », à l'Assemblée mais aussi dans son lit: il n'est pas jusqu'aux positions choisies par les amants qui ne reflètent leur adhésion au nouveau régime. Mais ce discours politique passe désormais par le catalogue. Au lecteur avisé de repérer les postures conformes à la nouvelle situation du pays. Pour rendre leur message encore plus convaincant, ces manuels recourent très souvent à la figure d'Hercule. Héros invincible, il incarne avec l'efficacité d'un slogan les valeurs de l'érotique révolutionnaire : force, fougue, prouesse. Les manuels encensent ainsi la position dite "de l'Hercule », qui exige des amants de la puissance et des muscles solides. Dans Les Quarante manières de foutre, il s'agit de la trentequatrième «manière »:

La femme se met à califourchon sur son fouteur assis sur une chaise. Elle prend son vit à pleine main, le branle jusqu'à ce que, gonflé par les esprits vitaux, il lève une tête altière. Ensuite elle le place dans son con et passe ses bras autour du col de son amant, qui lui passe les siens sous les cuisses et prend le haut de ses fesses, puis se lève tout debout et la fout ainsi en se promenant. Pour essayer de cette manière, il faut être fort de reins : c'est la plus fatigante. Petits-maîtres, Hercule ainsi foutait. C'est assez vous dire que c'est au-dessus de vos forces. ${ }^{23}$

L'objectif pédagogique est ici double: saluer l'énergie des acteurs de la Révolution, et dénoncer l'impuissance des «petitsmaîtres ». Le message s'appuie sur une confrontation d'images : au torse musculeux d'Hercule s'oppose l'organisme affaibli des courtisans «mondains », ces survivants des boudoirs de l'Ancien Régime. Les gravures dont ces ouvrages sont le plus souvent illustrés accentuent encore ce contraste, tirant le corps révolutionnaire vers un athlétisme idéalisé ${ }^{24}$.

23. Les Quarante manières de foutre, dédiées au clergé de France, in CEuvres anonymes du XVIII' siècle, «L'Enfer de la B. N.» (Paris, Fayard, 1986), t. 3, p. 390.

24. «Le but est de produire des champions, et pas seulement des techniciens. Au-delà de la simple maîtrise, c'est l'exploit qu'il faut viser. Dans tous les manuels de postures, le lecteur est frappé par la surenchère athlétique des leçons, que traduisent également les gravures : muscles tendus par l'effort, corps tordus, membres engagés dans une gymnastique qui paraît épuisante, tout y dit la force, la vigueur. La précision anatomique dans les descriptions et les illustrations 
Ce combat symbolique entre Hercule et l'homme galant se retrouve dans un autre manuel publié lui aussi en 1790, La Rocambole de la fouterie. Le titre complet ressuscite l'arsenal mythologique de la prouesse : ces nouveaux travaux d'Hercule chargent le héros de redonner force et vigueur à la sexualité des patriotes. Sa dernière hydre, c'est l'Ancien Régime et ses acteurs privilégiés :

Les petits-maîtres, ces pygmées en galanterie, et toutes ces femmes qui affectent la délicatesse du genre nerveux, vont sans doute se récrier sur des obligations pénibles; j'avais prévu d'avance leurs objections ; et, pour toute réponse, je me contenterai de leur exposer que les plaisirs que je vais décrire ne sont pas faits pour eux; qu'il faut pour les goûter une constitution mâle et vigoureuse ; que ce n'est point à des débiles et des efféminés que j'offre mes opuscules en galanterie, que c'est à des hommes sains et dispos, à des tempéraments de la bonne trempe, enfin à des hommes qui puissent remplir la tâche que le titre de mon ouvrage leur impose. Je veux moins démontrer les jouissances que l'on peut éprouver relativement à ces facultés, que celles que la nature, dans toute son énergie, est susceptible de procurer à ceux chez qui elle n'est point altérée. Je veux enfin saisir le plaisir dans sa source et non le saisir par ses ramifications. [éd. cit., p. 193]

Le narrateur exclut donc de son texte les corps faibles ou diminués : ni personnages ni lecteurs, ces organismes fatigués n'auront aucune place dans ce bréviaire, pas plus qu'ils n'en trouveront dans la France révolutionnée. L'exclusion s'accompagne pourtant d'un projet constructif, former des Hercules au lit et des patriotes pour le régime :

Venez entendre les leçons du plaisir, je professe aujourd'hui le grand art de la fouterie ; j'enseigne les moyens de se procurer des jouissances factices au défaut des naturelles; je donne des préceptes galants sur toutes les manières d'alambiquer le plaisir par les attouchements, les frottements, les titillations réitérées, et en général par tous les stimulants quelconques qui ont la vertu d'exciter, de provoquer les sens et de les réveiller de l'engourdissement où les tiennent la mollesse et l'inaction. Je forme des Hercules, des hommes extraordinaires enfin, en développant dans l'être organisé les facultés viriles de l'humaine nature. [p. 191]

excède le réalisme morphologique et finit par produire des corps trop humains, monstrueux à force de puissance et de flexibilité. L’art qui se pratique dans ces arts de foutre outrepasse les données "naturelles" sur lesquelles il se fonde pourtant: on y apprend la démesure, on y pratique l'excès, on y recherche l'exploit. La force génitale est exaltée: au lieu d'attirer l'homme vers le bas et de le rappeler à son animalité, elle le hisse vers le haut, le fait accéder au sublime », Stéphanie Loubère, «Hercule foutromane : l'hérö̈sme amoureux au tournant du XVIII" siècle », Orages n' 2, mars 2003, p. 75. 
Un parallèle s'établit alors entre la formation du corps et la conscience politique : maîtriser les «leçons du plaisir » permet l'émergence d' ' hommes extraordinaires », amants hors pair mais aussi, et surtout, citoyens modèles au service de la nation. Et le plaisir se mesure à l'aune de nouvelles valeurs, comme l'élan du naturel :

L'auteur de la nature, en travaillant à nous faire éprouver des sensations voluptueuses, a voulu, pour la félicité de ses créatures, que le plaisir se trouvât le plus simplement possible, et qu'il ne consistât point dans ces recherches minutieuses qui prouvent plutôt l'ouvrage de l'art perfectionné que l'émanation directe de ses volontés. C'est pourquoi I'homme privé de toute instruction, qui n'a pour toute théorie que le seul instinct dont il est doué, sait, malgré son ignorance, trouver la voie du plaisir avec une femme aussi peu faite que lui aux exercices de ce genre : tout le monde est d'accord que la nature suffit pour donner des leçons à l'être le plus insensible, c'est un axiome si généralement reconnu qu'il n'est pas nécessaire, pour démontrer son authenticité, de l'appuyer d'aucune preuve. [p. 193]

Cette sexualité tonique s'incarne aussi dans l'omniprésence du mot «foutre » et de ses dérivés pendant la Révolution; le verbe désigne dans le même geste l'ardeur érotique et la vigueur du corps : «Le mot attire la foudre, il est foudre lui-même » ${ }^{25}$. Les titres des pamphlets l'utilisent presque tous pour sa force suggestive: Les quarante manières de foutre, La Rocambole de la fouterie, L'Art de foutre en quarante manières, Requête et décret en faveur des putains, des fouteuses, Hercule fouteur, $L$ 'Écho foutromane, autant d'exemples qui montrent l'extension du mot et sa puissance dérivative. La sexualité révolutionnaire trouve en lui un parfait miroir lexical : rapide, fertile, énergique, il renouvelle lui aussi le corps de la langue au tournant du siècle.

Cette lecture politique se durcit après 1792 : suivant en cela la législation révolutionnaire qui se radicalise, en particulier à l'égard des nobles et des émigrés, elle se teinte d'une violence nouvelle. Leur corps n'est plus seulement malade ou coupable, il devient monstrueux. Les pamphlets adoptent alors un ton plus virulent : l'heure n'est plus à la représentation critique, mais à l'appel au meurtre. Les Travaux d'Hercule offraient déjà, en 1790, les premiers signes de ce changement d'optique. Le catalogue s'achevait sur la description de la posture la plus citoyenne, « la

25. Patrick Wald Lasowski, La Science pratique de l'amour, p. 12. 
bonne manière ou celle du bougrement patriotique père Duchesne » :

Il faut, sacré nom d'un million de cons vérolés ! pour foutre à la manière bougrement patriotique de l'énergique père Duchesne, empoigner par la cotte une garce à cul, et lui ouvrir, sacré triple nom d'une vieille tétasse! sa vilaine foutue fressure de vache, en écartant avec le pouce et les autres doigts les sacrées babines de sa poche à poulains, sans oublier, surtout, de lui faire bailler le cardinal. [...] Quand il commence à lever sa sacrée vénérable tête cicatrisée par la pierre infernale, le bistouri et le scalpel, on dit à la sacrée garce : "Ah ça, fais belle motte, putain, c'est le père Duchesne qui va te foutre une culotte à la bonne manière, entendu ? Si je ne te fous pas un vit fabriqué comme le tuyau du poêle des Invalides, je te permets de m'appeler le plus grand bande-à-l'aise de tous les aristocrates." [éd. cit., p. 206]

L'écriture se charge ici d'une violence qui n'est pas sans rappeler celle du journal extrémiste d'Hébert. Les appels à la délation et les exigences toujours plus fortes de ce périodique informent la description : tout y est radicalisé, le ton comme le vocabulaire, si bien que l'érotisme lui-même disparaît sous le flot des injures. Le texte prend alors ses distances avec cette parole du Père Duchesne, en utilisant des guillemets, absents des pages précédentes. Mais il n'escamote pas le durcissement qui s'amorce, et qui trouve dans les années de Terreur un contexte particulièrement propice. La volonté d'éradiquer l'Ancien Régime passe alors par la suppression, au sens propre, de tous ses corps : sociaux, puisque l'aristocratie est bel et bien hors-la-loi, et biologiques puisque la guillotine semble le prolongement concret de ce projet. Avant les exécutions en nombre, et qui symboliquement mutilent le corps des victimes, plusieurs figures du pouvoir font l'objet d'attaques d'une rare sauvagerie. La princesse de Lamballe, pour ne citer qu'un des exemples les plus célèbres, meurt dans les massacres de septembre dans les pires conditions: sa tête promenée au bout d'une pique jusque sous les fenêtres de Marie-Antoinette, à qui elle a juré fidélité jusqu'au bout, n'est que l'ultime étape d'un programme de destruction du corps: violée, éviscérée, on lui arrache le cœur avant d'abandonner sur place sa dépouille décapitée. Cet acharnement témoigne, au-delà de la « haine du noble », d'une volonté de faire disparaître jusqu'au corps de l'ennemi : cette enveloppe charnelle qui constitue la première identité devient insupportable. La cruauté des sansculottes s'explique alors par une curiosité effroyable, qui pousse à percer le mystère de ce corps nimbé de privilèges et d'inconnu : maintenu pendant si longtemps dans le secret des cabinets, il 
doit désormais s'ouvrir au nom de la transparence qui s'installe. Mais ce corps mis à nu est aussi une incarnation de l'autre, de cette différence inadmissible pour l'égalitarisme révolutionnaire : la pulsion scopique dégénère alors en massacre, en exécution sauvage. Guillotiner, démembrer, éviscérer, retire définitivement au corps des élites son caractère sacré. L'exécution du roi marque dans ce sens une étape décisive, et un traumatisme sans précédent pour les spectateurs de la scène. En coupant la tête du monarque, la Révolution ajoute à la métaphore une acception sanglante : pour éradiquer un corps collectif, il faut amputer le corps individuel. $L a$ Ligue aristocratique présente l'aristocrate comme un vampire qui fomente en secret la ruine de la Révolution:

Oui, monstres, votre confédération sordide et assassine, fait plus : elle accapare nos grains; et les broie avec le poison pour nous faire acheter la mort en nous survendant la vie. Vous étiez les moteurs, les complices de l'exécrable Foulon et de l'odieux Savigny; comme eux vous mériteriez qu'on arrachât vos cœurs corrompus, et qu'on traînât dans la fange les corps vénéneux que vous avez déjà plongés dans les vices. 26

Il faut non seulement le supprimer, mais lui arracher le cœur, ouvrir son corps, et violer jusqu'à sa dernière intimité. Cette violence s'en prend plus particulièrement aux femmes, et aux femmes de pouvoir. Les travaux de Chantal Thomas ont souligné la puissance des fantasmes qui auréolent le personnage de la reine : accusée d'être autre géographiquement - l'Autrichienne politiquement - l'ennemie du régime - et sexuellement - la tribade, Marie-Antoinette meurt surtout à cause des tares qu'on lui prête. La frontière entre la propagande et la réalité s'estompe alors dangereusement : les révolutionnaires croient à ce qu'ils lisent, et confèrent au corpus des pamphlets une force sans précédent. Décapitant la reine, ils ont la ferme illusion de mettre à mort l'érotique décadente de l'Ancien Régime, avec son narcissisme, son tribadisme, et sa « fureur utérine ». Le corpus, miroir du corps, achève de boucler les extensions, si radicales fussentelles, de la métaphore.

La Révolution associe donc définitivement la sexualité et la politique. Le corps établit entre les deux domaines une passerelle sans précédent, même si elle s'appuie également sur le fonctionne-

26. La Ligue aristocratique, ou les Catilinaires françaises, par un membre du Comité patriotique du Caveau du Palais-Royal, in Chantal Thomas, La Reine scélérate, Marie-Antoinette dans les pamphlets (Paris, Seuil, 1989), p. 211. 
ment du système dans son ensemble. L'Ancien Régime appelait une érotique narcissique là où la liberté restaure, en plus de la démocratie, la force et l'énergie. Au-delà des corps, ce sont deux organisations qui s'opposent, et qui déterminent chacune un mode de séduction spécifique. C'est du moins le sens qui se dégage en 1791 d'un texte décisif sur ce plan, Julie philosophe ou le bon patriote. La narratrice voyage à travers toute l'Europe, compare les différents systèmes politiques mais aussi les visages de la séduction qu'elle rencontre : révolutionnaire, Julie est aussi courtisane, et apparaît à ce titre comme un personnage emblématique de la littérature de cette époque. Comme elle, elle croise les domaines et les différents corps qui s'affrontent. En Angleterre, elle loue donc sans surprise le récent avènement de la liberté. Ce pays a vaincu le despotisme, même si cette révolution eut un prix érotique, la perte des galanteries et de toute forme de raffinement :

C'est une chose remarquable que plus les peuples sont libres, moins ils sont galants, moins ils connaissent cette fleur de politesse et d'urbanité, cette manière fine et spirituelle de courtiser les femmes, si bien pratiquée chez nous. [...] Mais comme l'esprit de liberté n'exclut point la sensibilité qu'au contraire, il fortifie, il exalte ce principe de toute vertu et lui donne ce caractère mâle et élevé qui nous porte à des actions sublimes et héroïques, si un homme est sensible aux charmes d'une femme, cet intérêt se manifeste d'une manière décidée, rapide, brusque et violente; l'expression de son amour est hardie comme sa pensée ; il ignore ces lieux communs amoureux, ces préliminaires galants qu'on connaît si bien en France, et comme il ne sent rien faiblement, qu'il n'est point accoutumé à former des désirs qu'il ne puisse satisfaire, il marche sans détour à son but, et voir, désirer, jouir n'est presque pour lui qu'une seule et même chose. Français, qui venez de secouer les fers du despotisme, prenez garde de perdre cette agréable galanterie, cet heureux talent de courtiser le sexe qui vous distingue. En devenant libres, vous en deviendriez moins aimables. ${ }^{27}$

Le texte élargit donc au « macrocosme » politique les représentations associées au corps privé : si l'aristocrate est biologiquement corrompu, la faute en incombe davantage à l'absence de liberté qu'à une défaillance de son «groupe ». À l'inverse, le patriote doit sa fougue à la proclamation de la liberté plus qu'aux vertus de son seul organisme. La France entre ainsi, en 1791,

27. Julie philosophe ou le bon patriote, histoire à peu près véritable d'une citoyenne active qui a été tour-à-tour agent et victime dans les dernières révolutionis de la Hollande, de Brabant et de France, 1791 (Paris. Tchou, 1968), p. 205. 
dans une ère nouvelle : démocratique, mais moins érotique. Elle montre en tous cas, par ce sursaut sublime, que la fatalité de l'essoufflement peut être enrayée :

Oh ! nation libre et heureuse, nation pleine d'énergie, vous êtes, quoi qu'on en dise, la première nation de l'Europe; vous l'étiez du moins avant la journée du 14 juillet 1789, mais les Français, par le noble courage qu'ils ont déployé, par cet amour de la liberté qui a fructifié en eux d'une manière aussi rapide qu'étonnante, et les a portés à des entreprises hardies et presqu'inconcevables, les Français ont franchi en une journée l'intervalle qui les séparait encore de vous. Vos égaux par le génie et les lumières, ils le sont devenus tout d'un coup par l'énergie ; ce pas a été un pas de géant ; ç'a été un effort sublime, un passage rapide du néant à l'existence, puisqu'on peut dire que l'esclavage est le néant moral de l'homme, et la liberté son existence active. [p. 221]

Le message à transmettre, ce qui fait au tournant du siècle la réputation de la France, se charge alors de valeurs nouvelles: à la maitrise du corps - le stéréotype de l'art de jouir français s'ajoute désormais le triomphe de la liberté.

STÉPHANIE GENAND 